\title{
Invariance of the relativistic one-particle distribution function.
}

\author{
F. Debbasch \\ Université Paris 6 - C.N.R.S., L.R.M. (E.R.G.A.), \\ Tour 22-12, $4^{\text {ème }}$ étage, boîte 142 \\ 4 place Jussieu, 75252 Paris Cedex 05, France \\ J.P. Rivet \\ C.N.R.S., Laboratoire G.D. Cassini, Observatoire de Nice, \\ F-06304 Nice Cedex 04, France \\ W.A van Leeuwen \\ Instituut voor Theoretische Fysica \\ Valckenierstraat 65, 1018 XE, Amsterdam, The Netherlands \\ Physica A, 301, pp. 181-195, 2001
}

\begin{abstract}
The one-particle distribution function is of importance both in non-relativistic and relativistic statistical physics. In the relativistic framework, Lorentz invariance is possibly its most fundamental property. The present article on the subject is a contrastive one: we review, discuss critically, and, when necessary, complete, the treatments found in the standard literature.
\end{abstract}

PACS numbers: 03.30.+p, 05.20.Dd

Keyword: distribution function, special relativity. 


\section{Introduction}

One of the most important and fruitful concepts in statistical physics is the concept of phase-space. If one restricts the analysis to the non-quantum level, the state of every Galilean system consisting of $N$ point-like particles can, at any time, be represented by one point in a $6 N$-dimensional phase-space [1]. The statistical behavior of such a system can then be described by an evolution equation for a distribution function, often called phase-space density and notated $\rho\left(t, \boldsymbol{r}^{N}, \boldsymbol{p}^{N}\right)$, defined, at fixed time $t$, on this $6 N$-dimensional phase-space spanned by the $3 N$ positions $\boldsymbol{r}^{N}=\left(\boldsymbol{r}_{1}, \ldots, \boldsymbol{r}_{N}\right)$ and the $3 N$ momenta $\boldsymbol{p}^{N}=\left(\boldsymbol{p}_{1}, \ldots, \boldsymbol{p}_{N}\right)$ of the $N$ particles. In many physically interesting cases, however, the particles which constitute the system can be considered as weakly interacting only, and it then makes sense to introduce the concept of a one-particle distribution function, often notated $f(t, \boldsymbol{r}, \boldsymbol{p})$, and defined - at any instant $t$ - on a 6-dimensional (i.e, the one-particle) phase-space [2].

In relativistic physics, the concept of a one-particle distribution function $f$ is also widely used, and it seems even more important to relativistic statistical mechanics than its Galilean homologue is to Galilean statistical physics [3, 4]: when electromagnetic interactions are included, it does not seem possible to introduce, at a relativistic level, an analogue for the Galilean $N$ particle distribution function $\rho\left(t, \boldsymbol{r}^{N}, \boldsymbol{p}^{N}\right)$, since the transmission of electromagnetic signals can no longer be treated as if they occurred instantaneously. Therefore, the concept of one-particle distribution has become one of the cornerstones of non-quantum relativistic statistical mechanics: in practice, the one-particle distribution function $f(t, \boldsymbol{r}, \boldsymbol{p})$ is all one has in relativity.

The natural expression of the particle four-current in terms of the oneparticle distribution function [5] strongly suggests that the latter quantity has to be a Lorentz-scalar for the theory to be consistent within a relativistic framework. However, the literature on the notion of relativistic one-particle distribution offers, when submitted to a critical reading, a rather confusing perspective. Indeed, various authors differ on the very definition of the concept of distribution function and, consequently, on what should be proved and what has to be put in by hand. Many authors start from a non-manifestly covariant definition of the one-particle distribution $f$ that is formally identical with the usual non-relativistic one, and then the task remains to show that such a function is invariant under a change of reference-frame, i.e., that it is a scalar (see, e.g. [6], [7] and [5]). To achieve this goal there are, on the one hand, approaches which are a kind of relativistic extensions of the non- 
relativistic ones. They, in turn, fall into two basically different types: one type based on the so-called invariance of the volume element in phase-space [6, 7], the other type based on a manifestly covariant rewriting of the most general microscopic definition of the one-particle distribution $f$ in terms of mean-values with the help of Gibbs-ensemble averages over delta functions [8, 5]. What remains puzzling here is that both types of approach have very different physical and mathematical bases, and do not seem to rely on the same kind of argumentation at all.

On the other hand, there is a more axiomatic approach to the problem of introducing a relativistically invariant distribution function $f$; this other approach starts from a concept that is manifestly relativistic and Lorentzinvariant, namely the distribution function $f_{w}$ for the number of particles world-lines that cross an arbitrary space-like hyper-surface in space-time (see, e.g., [3] and [4]). The authors who use such a concept derive from it the usual concept of a particle distribution-function and have then little difficulty in proving that the standard particle distribution is also frame-independent. But a direct, microscopic definition of the distribution function for worldlines, comparable to the standard one for the particle distribution given in terms of mean-values of delta-functions over some Gibbs-ensemble, has not yet been given in the literature; as a consequence it has never been proven that such a world-line distribution function even exists nor that it is frameindependent. Both assertions are indeed treated as postulates and this is a rather uncomfortable situation, especially considering the fact that other authors, as mentioned earlier, seem to be able to establish as a theorem the fact that the particle distribution function is a scalar without having to introduce the new concept of world-line distribution.

Our aim is to revisit these issues and to shed some new light on them. Since the special and general relativistic discussions exhibit mathematical and physical difficulties which only partly overlap, we thought it would make things clearer to actually separate the special and general theory, and to present their treatments in two separate publications. The present article is, therefore, devoted to the special relativistic case only, while its following companion starts where this one stops and addresses the general relativistic situation.

In this article, we start from the standard definition for the one-particle distribution function in phase-space. The crucial issue is then to determine whether or not it is possible to establish, by direct reasoning, that the oneparticle-distribution function, so defined, is frame-independent. 
In section 2, we review the first type of proof, based on the so-called invariance of the one-particle phase-space volume under Lorentz-transformation [6, 7]. We show in a mathematically rigorous manner that the phase-space volume is not Lorentz-invariant; we also explain why this does not contradict the fact that the one-particle distribution in phase-space may be Lorentzinvariant and that the whole argument is just inconclusive. In section 3, we analyze the proof originally developed by de Groot and Suttorp [8], of which a pedagogical presentation can also be found in the book by de Groot, van Leeuwen and van Weert [5]. This proof is based on the most general definition of the distribution function in phase-space. As such, it makes use of the concept of an ensemble average and presupposes this procedure to be covariant. We actually show that this procedure is not a priori covariant because it relies on the concepts of macro- and microstates, which are shown not to be Lorentz-invariant. We therefore introduce the new covariant concepts of macroscopic and microscopic 'histories' and define, in a manifestly Lorentzinvariant way, statistical ensembles. The average over these new ensembles is de facto a scalar procedure and it provides a new definition of the oneparticle distribution function which ensures that this function is definitely a Lorentz-scalar. We then show that, contrarily to what might have been expected, the average over these covariant ensembles actually comes down to the usual average over states and that the usual relativistic one-particle distribution function is therefore, indeed, a Lorentz-scalar; this completes the validation of the proof of [5].

In section 4, we discuss the notion of one-particle distribution function for particles world-lines crossing an arbitrary hypersurface in space-time. We prove that this notion only makes sense because the particle-distribution in phase-space is a Lorentz-scalar. In other words, if one chooses the axiomatic approach to relativistic kinetic theory, postulating that the world-line distribution function exists is tantamount to postulating that the one-particle distribution function in phase-space is frame-independent. Moreover the world-line distribution function turns out to be identical with the standard particle distribution. Finally, in section 5, we give an overview of our results and we discuss them in some detail. 


\section{Earlier attempts to define the one-particle distribution function}

The usual definition of the one-particle distribution function in special relativity is not completely satisfactory. Strictly speaking, it is even wrong. It is one of the purposes of this section to analyze in detail what happens exactly when one counts particles in different systems of reference, and to relate our results to the corresponding ones found in the standard literature.

The final and disappointing conclusion of this section will be that the usual approaches, based on the so-called Lorentz-invariance of the phasespace volume-element, have all failed if one takes them really seriously. It is our goal to develop a better approach. This is the subject of the next section, section 3. In order to make the failure of the earlier attempts as clear as possible we first follow, in the present section, the usual approach as far as possible.

Let $\mathcal{R}$ be an arbitrary Lorentz frame, with respect to which we want to study a gas of particles. Let

$$
d N(t, \boldsymbol{x}, \boldsymbol{p})
$$

be the number of particles which, at time $t$, in $\mathcal{R}$, have positions and momenta in the intervals $(\boldsymbol{x}, \boldsymbol{x}+d \boldsymbol{x})$ and $(\boldsymbol{p}, \boldsymbol{p}+d \boldsymbol{p})$, respectively. Let us denote the phase space volume elements corresponding to these intervals by $d^{3} x$ and $d^{3} p$.

Now, the one-particle distribution function $f(t, \boldsymbol{x}, \boldsymbol{p})$, at time $t$, in $\mathcal{R}$, is defined by the relation:

$$
d N(t, \boldsymbol{x}, \boldsymbol{p})=f(t, \boldsymbol{x}, \boldsymbol{p}) d^{3} x d^{3} p .
$$

Obviously, the distribution function $f$ has the dimensions of a density in position and momentum space. We now want to prove that the function $f$, defined via the equation (2), is a Lorentz scalar. To that end we introduce, next to the reference system $\mathcal{R}$, a new system of reference, $\mathcal{R}^{\prime}$ which moves with three-velocity $\boldsymbol{v}$ with respect to $\mathcal{R}$. For reasons of simplicity, we choose $\boldsymbol{v}$ parallel to $\boldsymbol{p}$, the momentum of the particles within $d^{3} x d^{3} p$ on which we are now focusing our attention. Furthermore, we choose the $x$-axis of $\mathcal{R}$ and $\mathcal{R}^{\prime}$ both parallel to $\boldsymbol{v}$.

Because changes occur only in the $x$-directions, the $y$ - and $z$-components of position and momentum variables remain unchanged under the Lorentz 
transformations relating the reference systems $\mathcal{R}$ and $\mathcal{R}^{\prime}$. We have:

$$
\begin{aligned}
c t^{\prime} & =\gamma(v)\left(c t-c^{-1} v x\right) \\
x^{\prime} & =\gamma(v)(x-v t) \\
y^{\prime} & =y \quad, \quad z^{\prime}=z,
\end{aligned}
$$

with $v=|\boldsymbol{v}|$ is the norm of $\boldsymbol{v}=(v, 0,0)$, and where $\gamma$ is the 'dilatation factor'. The latter is defined, for arbitrary $\boldsymbol{v}$, by :

$$
\gamma(v):=\frac{1}{\sqrt{1-v^{2} / c^{2}}} .
$$

¿From (3)-(15) we find :

$$
\begin{aligned}
c d t^{\prime} & =\gamma(v)\left(c d t-c^{-1} v d x\right) \\
d x^{\prime} & =\gamma(v)(d x-v d t) \\
d y^{\prime} & =d y, d z^{\prime}=d z
\end{aligned}
$$

Since, by hypothesis, the particles under consideration occupy, in the reference frame $\mathcal{R}$, a purely spatial element $d^{3} x$ characterized by:

$$
t=\text { constant }
$$

we have, in $\mathcal{R}$ :

$$
d t=0
$$

Hence, eqs. (7)-(8) reduce to :

$$
\begin{gathered}
d t^{\prime}=-\gamma(v) c^{-2} v d x \\
d x^{\prime}=\gamma(v) d x
\end{gathered}
$$

En passant, we note that equation (13) explains the name dilatation factor for $\gamma(v)$. From (9) and (13) we find:

$$
d^{3} x^{\prime}=\gamma(v) d^{3} x
$$

We now come to the transformation in momentum space. If we suppose, for a moment, that $p^{0}$, on the one hand, and $p^{x}, p^{y}, p^{z}$ on the other hand, are independent variables, we have:

$$
\begin{aligned}
& {p^{\prime}}^{0}=\gamma(v)\left(p^{0}-c^{-1} v p^{x}\right) \\
& p^{\prime x}=\gamma(v)\left(p^{x}-c^{-1} v p^{0}\right) \\
& p^{\prime y}=p^{y}, p^{\prime z}=p^{z}
\end{aligned}
$$


and thus :

$$
\begin{aligned}
d p^{\prime 0} & =\gamma(v)\left(d p^{0}-c^{-1} v d p^{x}\right) \\
d p^{\prime x} & =\gamma(v)\left(d p^{x}-c^{-1} v d p^{0}\right) \\
d p^{\prime y} & =d p^{y}, \quad d p^{\prime z}=d p^{z} .
\end{aligned}
$$

However, $p^{0}$ and $\boldsymbol{p}$ are not independent. From the normalization of the fourmomentum $p^{\mu} p_{\mu}=m^{2} c^{2}$ we find:

$$
p^{0^{2}}=m^{2} c^{2}+p^{x 2}+p^{y 2}+p^{z 2}
$$

or

$$
d p^{0}=\frac{1}{p^{0}}\left(p^{x} d p^{x}+p^{y} d p^{y}+p^{z} d p^{z}\right) .
$$

Substituting (22) into (19) we obtain :

$$
d p^{\prime x}=\gamma(v)\left(1-\frac{v}{c} \frac{p^{x}}{p^{0}}\right) d p^{x}-c^{-1} v \gamma(v) \frac{p^{y} d p^{y}+p^{z} d p^{z}}{p^{0}}
$$

or, equivalently, using (15) :

$$
d p^{\prime x}=\frac{p^{\prime 0}}{p^{0}} d p^{x}-\frac{v}{c} \gamma(v) \frac{p^{y} d p^{y}+p^{z} d p^{z}}{p^{0}} .
$$

Hence, using also (20) :

$$
d p^{\prime x} \wedge d p^{\prime y}=\frac{p^{\prime p}}{p^{0}} d p^{x} \wedge d p^{y}-\gamma(v) \frac{v}{c} \frac{p^{z}}{p^{0}} d p^{z} \wedge d p^{y},
$$

since the term $d p^{\prime y} \wedge d p^{y}=d p^{y} \wedge d p^{y}$ cancels. Similarly, we find:

$$
d p^{\prime x} \wedge d p^{\prime y} \wedge d p^{\prime z}=\frac{p^{\prime 0}}{p^{0}} d p^{x} \wedge d p^{y} \wedge d p^{z}
$$

or

$$
\frac{d p^{\prime x} \wedge d p^{\prime y} \wedge d p^{\prime z}}{p^{\prime 0}}=\frac{d p^{x} \wedge d p^{y} \wedge d p^{z}}{p^{0}}
$$

or, equivalently:

$$
\frac{d^{3} p^{\prime}}{p^{0}}=\frac{d^{3} p}{p^{0}}
$$


a well-known result.

In short, we find that (14) and (28) imply:

$$
d^{3} x^{\prime} d^{3} p^{\prime}=\gamma(v) \frac{p^{\prime 0}}{p^{0}} d^{3} x d^{3} p .
$$

An alternative form for ${p^{\prime}}^{0}(\underline{15})$ is :

$$
p^{\prime 0}=\gamma(v) p^{0}\left(1-c^{-1} v \frac{u}{c}\right) .
$$

where $u$ is the norm of the particle three-velocity $c \boldsymbol{p} / p^{0}=(u, 0,0)$. With (30) we find from (29) :

$$
d^{3} x^{\prime} d^{3} p^{\prime}=\frac{1-v u / c^{2}}{1-v^{2} / c^{2}} d^{3} x d^{3} p,
$$

where we used the definition (6) of $\gamma(v)$. We did not encounter the result (31) in the literature. In the particular case in which the arbitrary reference frame $\mathcal{R}^{\prime}$ coincides with the rest- or comoving-frame of the particles which move with momentum $\boldsymbol{p}$ with respect to $\mathcal{R}$, we have:

$$
u=v \text {. }
$$

Let us denote this particular system $\mathcal{R}^{\prime}$ by $\mathcal{R}^{*}$, and the position and momentum of particles in this particular co-moving system of reference by $x^{*}$ and $p^{*}$. We then find from (31) and (32) :

$$
d^{3} x^{*} d^{3} p^{*}=d^{3} x d^{3} p
$$

Hence, $d^{3} x^{*} d^{3} p^{*}$ is a scalar. However, contrarily to general belief, the phase space element $d^{3} x d^{3} p$ is not a Lorentz scalar, as is seen from (31). Let us digress a little bit on this point.

Equation (14) is a result valid for any Lorentz transformation, from one system of reference to another, arbitrary system of reference. In particular, we thus have:

$$
d^{3} x^{*}=\gamma(u) d^{3} x
$$

where $u$ is the velocity $\mathcal{R}^{*}$ with respect to $\mathcal{R}$. Hence, combining (14) and (34), we have:

$$
d^{3} x^{*}=\frac{\gamma(u)}{\gamma(v)} d^{3} x^{\prime}
$$


This relation is not always found in the existing literature. In the textbook 'The classical Theory of Fields', Landau and Lifschitz claim (section 10) that:

$$
d^{3} x^{*}=\gamma(u) d^{3} x^{\prime}
$$

basing themselves on (34) only, and thus forgetting the step leading to eq. (14). Misner, Thorne and Wheeler, in their textbook 'Gravitation', in Box 22.5, derive (33) and then conclude that the six-dimensional phase space element is invariant, which it is not, as implied by (31).

The point missed in most treatments encountered in the literature is that, in any given reference-frame, the volume elements which enter definition (2) for the distribution- function have to be considered at a fixed time in this reference-frame. In other words, the points of the volume element $d^{3} x$ in some system of reference $\mathcal{R}$ should be points on a hypersurface of the form $t=$ constant in that four-space $\mathcal{R}$. In our treatment this is made apparent by eq. (10); but, if this is so in one reference-frame $\mathcal{R}$, this is not so in any other reference-frame $\mathcal{R}^{\prime}$ : the same points in space-time occupy the space-volume $d^{3} x^{\prime}$ in $\mathcal{R}^{\prime}$ but do not belong to a hypersurface $t^{\prime}=$ constant, since $d t^{\prime} \neq 0$, as follows from eq. (12). It makes therefore no sense to count these points in $\mathcal{R}^{\prime}$ by using the one-particle distribution-function in $\mathcal{R}^{\prime}$, which is a priori suitable only for counting points on hypersurface of the form $t^{\prime}=$ constant (see again definition (2)).

The preceding considerations are all related to eq. (2), the defining relation of the one-particle distribution function $f(t, \boldsymbol{x}, \boldsymbol{p})$.

Being a number, the left-hand side of (2) is a scalar, which can be calculated in any reference system. This does not imply, however, that (2) can be used as the defining expression of the distribution function $f(t, \boldsymbol{x}, \boldsymbol{p})$ in an arbitrary system of reference $\mathcal{R}^{\prime}$. Let us again elaborate on this point with some more detail.

The inverses of the Lorentz transformations (33)-(6) and (15)-(17) may be used to express $t, \boldsymbol{x}$ and $\boldsymbol{p}$ in terms of $t^{\prime}, \boldsymbol{x}^{\prime}$ and $\boldsymbol{p}^{\prime}$. We may thus introduce $\tilde{f}$ defined by:

$$
\tilde{f}\left(t^{\prime}, \boldsymbol{x}^{\prime}, \boldsymbol{p}^{\prime}\right):=f\left(t\left(t^{\prime}, \boldsymbol{x}^{\prime}\right), \boldsymbol{x}\left(t^{\prime}, \boldsymbol{x}^{\prime}\right), \boldsymbol{p}\left(\boldsymbol{p}^{\prime}\right)\right) .
$$

With (37) and (31) we can reexpress (2) in the new coordinates $\left(t^{\prime}, \boldsymbol{x}^{\prime}, \boldsymbol{p}^{\prime}\right)$, which yields :

$$
f(t, \boldsymbol{x}, \boldsymbol{p}) d^{3} x d^{3} p=\tilde{f}\left(t^{\prime}, \boldsymbol{x}^{\prime}, \boldsymbol{p}^{\prime}\right) \frac{1-v^{2} / c^{2}}{1-u^{2} / c^{2}} d^{3} x^{\prime} d^{3} p^{\prime} .
$$


In the particular frame $\mathcal{R}^{*}$ co-moving with the particles with momentum $\boldsymbol{p}$ we have:

$$
f(t, \boldsymbol{x}, \boldsymbol{p}) d^{3} x d^{3} p=\tilde{f}\left(t^{*}, \boldsymbol{x}^{*}, \boldsymbol{p}^{*}\right) d^{3} x^{*} d^{3} p^{*} .
$$

Together with (2), eq. (39) suggests that $\tilde{f}\left(t^{*}, \boldsymbol{x}^{*}, \boldsymbol{p}^{*}\right)$ is the one-particle distribution function. However, this is not so, since the volume element $d^{3} x^{*}$ in (39) should be a part of a hyperplane $t^{*}=$ constant, which it is not, since $d t^{*} \neq 0$ [cf. eq. (12)].

More generally, the combination $\tilde{f}\left(t^{\prime}, \boldsymbol{x}^{\prime}, \boldsymbol{p}^{\prime}\right)\left(1-v^{2} / c^{2}\right)\left(1-u v / c^{2}\right)^{-1}$ is not the one-particle distribution function in $\mathcal{R}^{\prime}$, since $d^{3} x^{\prime}$ in (38) is not a part of the hyperplane $t^{\prime}=$ constant [again, see eq. (12)].

To summarize: the number $d N$ of eq. (2) indeed is a Lorentz scalar, as is generally stated. It is simply defined, in any reference system, as the number of particles which, at time $t$ in $\mathcal{R}$, occupy the phase-space element $d^{3} x d^{3} p$ centered around $(\boldsymbol{x}, \boldsymbol{p})$. This number, of course, can be evaluated in any Lorentz frame. Indeed, eq. (38) gives it expression in $\mathcal{R}^{\prime}$. However, $d N$ cannot be interpreted as a number of particles in $\mathcal{R}^{\prime}$. Therefore, it cannot be linked with the one-particle distribution function in that reference system. Thus the above calculations do not offer any clue as to what the correct distribution in $\mathcal{R}^{\prime}$ is, and the usual approach is inconclusive. This is the disappointing conclusion referred to in the introduction to the present section.

\section{The concept of macrohistory}

The only other direct proof that, in quite general a context, the relativistic one-particle distribution function in phase-space is a Lorentz-scalar, has been proposed in [8, 5]. We will first review rapidly the basics of this proof and then show that, in order for it to be fully consistent with the principles of Einstein's relativity, one must introduce the new concept of 'macrohistory' to replace the usual Galilean concept of 'macrostate'.

\subsection{A manifestly covariant expression for the distribu- tion function}

The basic idea behind the proof proposed in [8, 5] is to find a manifestly covariant expression for the distribution function in phase-space, without having to introduce the concept of world-line distribution function. To achieve 
this goal, the authors start from the standard, apparently frame-dependent definition :

$$
f(t, \boldsymbol{x}, \boldsymbol{p})=\left\langle\sum_{r} \delta\left(\boldsymbol{x}-\boldsymbol{x}_{r}(t)\right) \delta\left(\boldsymbol{p}-\boldsymbol{p}_{r}(t)\right)\right\rangle,
$$

where the sum extends to all particles in the system and the outer brackets 'indicate an ensemble average'. In the relativistic framework, the space and time degrees of freedom are but coordinates in what is called the fourdimensional space-time. It is therefore quite natural to introduce in relativistic statistical physics an 8-dimensional 'extended' one-particle phase-space, where a point has typically $\left(t, \boldsymbol{x}, p^{0}, \boldsymbol{p}\right)$ as coordinates. In such a phase-space, $p^{0}$ is understood as an independent quantity, not necessarily related to $\boldsymbol{p}$. Relativistic calculations are usually carried out more easily in this phasespace than in the traditional, 'more physical', 6-dimensional one; in the end, physical results can be recovered by restricting every equation to the massshell or, more precisely, to the sub-manifold of the mass-shell where $p^{0}>0$. In this spirit, de Groot, Suttorp, van Leeuwen and van Weert introduce another function, $\mathcal{F}$, defined over the 'extended' 8-dimensional one-particle phase-space by :

$$
\mathcal{F}\left(t, \boldsymbol{x}, p^{0}, \boldsymbol{p}\right)=2 \theta\left(p^{0}\right) \delta\left(p^{2}-m^{2} c^{2}\right) f(t, \boldsymbol{x}, \boldsymbol{p}),
$$

and prove that $\mathcal{F}$ is actually the ensemble average of a Lorentz-scalar. More precisely, their basic result is that $\mathcal{F}$ can be written:

$$
\mathcal{F}(t, \boldsymbol{x}, \boldsymbol{p})=c \sum_{\omega \in \Omega} w_{\omega} \sum_{i} \int \delta^{(4)}\left(x-X_{i \omega}\left(s_{i}\right)\right) \delta^{(4)}\left(p-P_{i \omega}\left(s_{i}\right)\right) d s_{i}
$$

where the index $i$ labels the particles in the system as well as their trajectories $\left(X_{i}\left(s_{i}\right), P_{i}\left(s_{i}\right)\right)$ in the extended phase-space, each trajectory being parameterized by its proper-time $s_{i}$. The sum over $\omega$ is the mathematical expression for the statistical averaging and $w_{\omega}$ represents the weight associated to each element $\omega$ in the statistical ensemble $\Omega$.

Since the product of the theta-function by the delta-distribution, as it appears on the right-hand side of (41) is a Lorentz-scalar, the authors conclude that the distribution function $f$ itself is Lorentz-scalar. This conclusion is indeed warranted, but only if the ensemble averaging procedure in itself does not change the transformation character (variance) of the quantity to which it is applied, which is the case if the statistical ensemble $\Omega$ and the coefficients $w_{\omega}$ are Lorentz-scalars. We will now show that this is not a priori 
the case for ensemble averages defined in the usual, Galilean way because the notions of macro- or microstate are not themselves covariant; we will therefore introduce the new concepts of macro- and microhistories and show with their help that, contrarily to what might have been expected, the traditional ensemble average is indeed a covariant operation.

\subsection{Relativistic covariant ensembles}

In Galilean statistical physics, ensembles are defined via the concept of macrostate. A macrostate of a system is defined by the values taken by certain macroscopic quantities or macroscopic fields. The nature and number of the macroscopic quantities are, to a certain extent, arbitrary, although some usual or natural choices exist. If, for instance, one wants to study a perfect gas out of equilibrium, macroscopic quantities often used to define a macrostate are the particle density field, the velocity field and an arbitrary 'thermodynamical' field, such as the temperature field. In some contexts, it seems useful to extend the number of fields, as is commonly done within the framework of Extended Thermodynamics theories.

To any macrostate of a given system correspond many systems that are macroscopically the same, but are different on a microscopic scale. A collection of systems that differ microscopically, but are identical macroscopically, is what is called an ensemble.

The essential point to realize is that, in Galilean Physics, the values of the various macrofields define the macrostate of the system at a given time in the reference frame where the statistical study is carried out. Similarly, microstates in Galilean Physics are always states of the system at a given time in a chosen reference frame. In the Galilean world, this poses no problem since time is invariant by a change of reference frame. But this is not the case anymore in the relativistic framework. To render this discussion more precise, let $A^{\mu \ldots \nu}$ be one of the macrofields, the value of which defines a macrostate of the system under consideration. Classical examples in relativistic hydrodynamics are the particle current density $j^{\mu}$, the entropy flux density $S^{\mu}$ and the stress-energy tensor $T^{\mu \nu}$. To begin with, let us choose to study the system in a given inertial frame $\mathcal{R}$. The macro-state of the system in $\mathcal{R}$ at time $t$ is defined by the values taken by the macrofield $A^{\mu \ldots \nu}$ and all other macrofields on the hypersurface of space-time $(t=$ const.). The macrostate of the system, at a fixed time $t$ in $\mathcal{R}$, is therefore defined by the 
collection of numbers

$$
\hat{A}^{\mu \ldots \nu}(\boldsymbol{x}):=A^{\mu \ldots \nu}(t, \boldsymbol{x}),
$$

The change of the macrostate under a Lorentz-transformation characterized by the tensor $\Lambda^{\alpha}{ }_{\mu}$ can be investigated by applying $\Lambda$ directly to (43). One obtains immediately:

$$
A^{\alpha \ldots \beta}\left(t^{\prime}, \boldsymbol{x}^{\prime}\right)=\Lambda^{\alpha}{ }_{\mu} \ldots \Lambda^{\beta}{ }_{\nu} \hat{A}^{\mu \ldots \nu}(\boldsymbol{x}),
$$

where $t^{\prime}$ and $\boldsymbol{x}^{\prime}$ are related to $t$ and $\boldsymbol{x}$ by the same Lorentz transformation. In particular, $t^{\prime}$ in (44) depends on both $t$ and $\boldsymbol{x}$ or, equivalently, on $t$ and $\boldsymbol{x}^{\prime}$. The variable $t^{\prime}$ is therefore not constant in (44), but varies with $\boldsymbol{x}^{\prime}$; consequently, (44) does not define a macrostate of the system in $\mathcal{R}^{\prime}$. In other words, the concept of macrostate is not Lorentz-invariant; indeed, specifying the macrostate of a system in a given inertial frame does not fix the macrostate for the same system in other inertial frames. From the preceding discussion, it should be clear that the same conclusion also applies to microstates. Since the usual ensemble average is an average over all microstates corresponding to a given macrostate, it is not therefore obvious that the procedure is Lorentz-invariant. To analyze further the situation, it is necessary to introduce the new concepts of macro- and micro- histories.

Since the root of the apparent problem lies in the fact that the concept of state is not Lorentz-invariant, the natural idea is to replace that very concept by another one which is Lorentz-invariant. Let us therefore introduce the concept of history and define the macrohistory of a system by the values taken by various macroscopical fields at every point in space-time where the system exists. In a given inertial frame $\mathcal{R}$, this typically amounts to fixing the value of any of the retained macroscopical fields at every point $x$ in $\mathbb{R}^{3}$ for any value of $t$. The concept of microhistory will be defined accordingly. It is clear from the discussion in the preceding paragraph that these new concepts are Lorentz-invariant, i.e., specifying the macro- or microhistory of a system in a given inertial frame is sufficient to determine the macro- or micro- history of the same system in any other inertial frame. If one then defines a relativistic statistical ensemble $\Omega$ as the collection of systems with microhistories $\omega$ that correspond - for a sufficiently short period of time in the local co-moving system - to one and the same macrohistory, the ensemble averaging procedure is, by construction, Lorentz-invariant and the distribution function defined by (401) is, therefore, indeed, a Lorentz-scalar. Hence, we 
suppose that there exists collections of systems that are microscopically different, but macroscopically identical for some limited amount of time. This seems not too unrealistic an assumption.

Let us now prove that the covariant ensemble average over histories gives back the results obtained by the usual average over states. In ordinary statistical mechanics, the macroscopical fields always obey deterministic equations and one can, in principle, given an inertial reference frame $\mathcal{R}$, reconstruct the whole macrohistory of the system from its macrostate at an arbitrary time $t_{0}$ in $\mathcal{R}$ through the evolution equations. On the other hand, the microscopic degrees of freedom may obey deterministic equations or stochastic equations. If these equations are deterministic, the covariant average over all microhistories corresponding to a given macrohistory should coincide with the usual ensemble average over all the microstates corresponding to the given macrostate at time $t_{0}$ in $\mathcal{R}$. Indeed, through the deterministic microscopic evolution equations, one can then reconstruct the whole microhistories of the system from its microstates in $\mathcal{R}$ at an arbitrary time. In other words, deterministic evolution equations establish a one-to-one correspondence between the histories and the states of the system at a given time in $\mathcal{R}$. Thus averaging over histories comes down in such cases to averaging over states. The matter is more complicated if the microscopic evolution equations are stochastic, typically involving some random 'noise' (for an example of stochastic process in the relativistic framework, we refer the reader to [9] and [10]). For a given realization of the noise i.e. 'freezing' the randomness, the stochastic evolution equations act as deterministic ones and it is then possible to establish a one-to-one correspondence between histories and states. Naturally, this correspondence depends on the chosen realization. Keeping this realization fixed for the moment, the average over histories again comes down to an average over states. To get a full ensemble average, one usually also averages over the various realizations of the noise. Obviously, this final average does not change the variance of the quantity to which it is applied. Thus, the total ensemble average, including the average over the realization of the noise, is a covariant operation in this case too.

To sum up these results: the notions of macro-and microhistories are necessary to prove that the usual ensemble-averages over states are indeed covariant operations. This result is not trivial because, contrary to the concept of history, the notion of state is not a covariant concept. 


\section{The concept of world-line distribution func- tion}

As we indicated in the introduction to this article, some authors introduce $a b$ initio a new object in relativistic kinetic theory, the so-called distributionfunction for the world-lines of the particles and consider it to be the fundamental relevant concept for relativistic situations. We would like, in this section, to prove that the existence of such a distribution is, somewhat counterintuitively, not trivial; we will notably show that the world-line distribution, as it is usually defined in the literature, exists only because the standard particle distribution function is a Lorentz-scalar. In other words, to assume from the start that the world-line distribution function exists comes down to assuming that the usual one-particle distribution in phase-space is a Lorentzscalar.

\subsection{Definition of the world-line distribution function}

Let $\Sigma$ be any space-like hypersurface in space-time and $d \Sigma^{\mu}$ its normal fourvector at point $M\left(x^{\mu}\right)$. Let also $d^{3} p$ be a volume element in momentum space, centered on a given three-momentum $\boldsymbol{p}$. The world-line distribution function $f_{w}$ at point $Q=(t, \boldsymbol{x}, \boldsymbol{p})$ is usually defined so that the number of world-lines $d N_{w}$ with momentum in the range $\left(\boldsymbol{p}, d^{3} p\right)$ that cross $d \Sigma^{\mu}$ in the direction of its normal is given by:

$$
d N_{w}=f_{w}(Q) p^{\mu} d \Sigma_{\mu} \frac{d^{3} p}{p^{0}}
$$

$p^{0}$ naturally is the time-component of the four-vector $p^{\mu}$ associated to $\boldsymbol{p}$ by equation (21). Since $d N_{w}, p^{\mu} d \Sigma_{\mu}$ and $d^{3} p / p^{0}$ are Lorentz-scalars, it follows from equation (45) that $f_{w}$, if it exists, is also a Lorentz-scalar. What makes definition (45) not trivial is that the hyper-surface $\Sigma$, aside from being spacelike, is arbitrary. In particular, in any given reference frame, one can choose to apply equation (45) to an hyper-surface which does not coincide with the constant-time hyper-surfaces of this frame. This is why $d N_{w}$ is not, in general, the number of particles present in some infinitesimal volume of the phase-space at a given time in the chosen reference frame, but a number of world-lines. 


\subsection{The reason why $f_{w}$ exists}

Let $Q$ be any point in the one-particle phase-space, $M$ its projection on the space-time manifold and $\mathcal{R}$, an arbitrary inertial frame. It is always possible to find a space-like hyper-surface $\Sigma$ which contains $M$ and the time-like normal vector of which, $d \Sigma$, has vanishing space-components at $M$ in $\mathcal{R}$ :

$$
d \Sigma_{\mu}=d^{3} x \delta_{\mu}^{0}
$$

Transcribed in this reference-frame, equation (45) reads:

$$
d W=f_{w}(t, \boldsymbol{x}, \boldsymbol{p}) d^{3} x d^{3} p .
$$

Since the surface element $d \Sigma$ is, by construction, a constant-time surfaceelement in $\mathcal{R}$, the number of world-lines $d W$ is also the number of particles which, in this frame, occupy at time $t$ the phase-space volume $d^{3} x d^{3} p$. This proves that, if it exists, $f_{w}$ acts as (and is) the standard one-particle distribution function in $\mathcal{R}$ at point $\mathrm{Q}$.

Since $\mathcal{R}$ is arbitrary, this also proves that the definition of $f_{w}$ only makes sense because the particle-distribution function is frame-independent, i.e., because it is a Lorentz-scalar. To phrase it slightly differently, if one has not yet proven that the one-particle distribution function is a Lorentz-scalar, assuming that $f_{w}$ exists comes down to assuming that the particle distribution function is a scalar. On the contrary, if one has proven (as was done in section (3) ) that the particle distribution is a Lorentz-scalar, one can then introduce the invariant world-line distribution, prove that it exists and use it as a particularly elegant tool in manifestly covariant calculations. Let us also note that the very concept of world-line distribution, as opposed to particle distribution, seems to imply the concept of statistical average over histories, as opposed to average over states. All this is naturally perfectly coherent. Indeed, we have just seen that the concept of world-line distribution function only makes sense because the particle distribution function is a Lorentz-scalar, and it was proven in section 3 that the most natural and general way to ensure that the particle distribution is a Lorentz-scalar is precisely to use covariant statistical ensembles that are actually not ensemble of states, but ensembles of histories. 


\section{Discussion}

In this article, we have given a fresh look at the notion of relativistic distribution function commonly used in relativistic kinetic theory. Let us sum up our main results. As already assumed by various authors, the standard Galilean definition for the one-particle distribution function in phase-space can be imported safely into the special-relativistic realm. It is then possible to prove that this distribution is a Lorentz-scalar. However, the two direct proofs that exist in the literature have been found wanting. The first one is based on the so-called invariance of the volume-element in one-particle phasespace; we have proven by direct calculation that, contrary to earlier claims made by various authors, this volume-element is not Lorentz-invariant, and the whole proof has been shown to rest on a misconception of the problem. The second proof, due to de Groot and Suttorp (and later on incorporated in de Groot's, van Leeuwen's and van Weert's book on relativistic kinetic theory), starts from the most general definition of the one-particle distribution-function. We have shown that, to be fully convincing, this proof needs the introduction of the new manifestly-covariant (relativistic) concepts of micro- and macrohistories. With these notions, new, covariant statistical ensembles can be introduced and the one-particle distribution function can be shown to be indeed a Lorentz-scalar. We have also revisited the axiomatic approach to relativistic kinetic theory, which starts by introducing the non-Galilean concept of distribution function for the world-lines crossing an arbitrary space-like hypersurface in space-time. We have shown that introducing this new concept $a b$ initio in the relativistic theory is tantamount to assuming axiomatically that the usual particle distribution in phase-space is frame-independent. Since this is a fact which can be proven, as can be clearly seen from the argument of the present article, it seems to us that assuming it from scratch is unnecessary. On the other hand, building on the scalar-nature of the one-particle distribution to construct the distribution function for world-lines is certainly interesting, since the world-line distribution function is a most useful tool in manifestly covariant calculations.

This article would not be complete without a mention of another proof that the relativistic one-particle distribution in phase-space is a Lorentzscalar. This proof [10] is actually rather particular because it has been given in the context of relativistic stochastic processes only, and more precisely

for the distribution function associated to the so-called relativistic OrnsteinUhlenbeck process, which is a toy-model of relativistic diffusion. As such, 
this proof makes extensive use of stochastic calculus. How this connects with the general proof envisaged in this article is not absolutely clear yet and we hope to shed further light on this question in a forthcoming publication.

As also mentioned in the introduction, this article tackled with the special relativistic situation only. The general relativistic case is addressed in the article companion to the present one; envisaging the problem in an arbitrary reference-frame naturally contributes to a deeper understanding of the simpler, special-relativistic case, where the discussion is restricted to inertial frames only.

ACKNOWLEDGMENTS : The authors wish to acknowledge fruitful discussions with Cécile Barbachoux.

\section{References}

[1] L.D. Landau and E.M. Lifschitz, Statistical Physics, Vol. 5, 3rd edition, Pergamon Press (1980).

[2] K. Huang, Statistical Mechanics, John Wiley and Sons (1963).

[3] J. Ehlers, General Relativity and Kinetic Theory in General Relativity and Cosmology, R.K. Sachs Ed., Academic Press (1971).

[4] W. Israel, in Relativistic Fluid Dynamics, eds. A. Anile and Y. ChoquetBruhat (Springer-Verlag, Berlin 1989).

[5] S.R. de Groot, W.A. van Leeuwen and Ch.G. van Weert, Relativistic Kinetic Theory (North Holland, Amsterdam 1980).

[6] L.D. Landau and E.M. Lifschitz, The Classical Theory of Fields, Vol. 2, 4th edition, Pergamon Press (1975).

[7] C.W. Misner, K.S. Thorn and J.A. Wheeler, Gravitation (W.H. Freeman and Co., New-York 1973)

[8] S.R. de Groot, L.G. Suttorp, Foundations of Electrodynamics (NorthHolland Publishing Company, Amsterdam, 1972).

[9] F. Debbasch, K. Mallick and J.P. Rivet, J. Stat. Phys. 88(3/4), p. 945, 1997. 
[10] C. Barbachoux, F. Debbasch and J.P. Rivet, The spatially onedimensional relativistic Ornstein-Uhlenbeck process in an arbitrary inertial frame, Eur. Phys. J. B, 19, pp. 37-47, 2001. 